\title{
FINDING A BOUNDARY FOR A MENGER MANIFOLD
}

\author{
A. CHIGOGIDZE
}

(Communicated by James E. West)

\begin{abstract}
We give a characterization of $k$-dimensional $(k \geq 1)$ Menger manifolds admitting boundaries in the sense of Chapman and Siebenmann.
\end{abstract}

In [5] Chapman and Siebenmann considered the problem of putting a boundary on a Hilbert cube manifold (similar problems in the cases of smooth and piecewise linear manifolds were considered in $[2,14]$; a parametrical version of the above problem was considered in [13]). It was proved there that if a $Q$-manifold $M$ satisfies certain minimal necessary homotopy-theoretic conditions (finite type and tameness at $\infty$ ), then there are two obstructions $\sigma_{\infty}(M)$ and $\tau_{\infty}(M)$ to $M$ having a boundary. The first one is an element of the group $\left\lfloor\left\{\widetilde{\mathscr{K}}_{0} \pi_{1}(M-A): A \subseteq M\right.\right.$ compact $\}$, where $\widetilde{\mathscr{K}}_{0} \pi_{1}$ is the projective class group functor. If $\sigma_{\infty}(M)=0$, then the second obstruction can be defined as an element of the first derived limit of the inverse system $\lim \left\{\mathscr{W} h \pi_{1}(M-A)\right.$ : $A \subseteq M$ compact , where $\mathscr{W} h \pi_{1}$ is the Whitehead group functor. Further, it was proved in [5] that the different boundaries that can be put on $M$ constitute a whole shape class and that a classification of all possible ways of putting boundaries on $M$ can be done in terms of the group $\lim _{\complement}\left\{\mathscr{W} h \pi_{1}(M-A): A \subseteq\right.$ $M$ compact\} .

In the present paper we carry out a similar program for the problem of putting boundaries on $\mu^{n+1}$-manifolds, where $\mu^{n+1}$ denotes the $(n+1)$-dimensional universal Menger compactum (a $\mu^{n+1}$-manifold $M$ admits a boundary if there exists a compact $\mu^{n+1}$-manifold $N$ such that $M=N-Z$, where $Z$ is a $Z$-set in $N$; in this case we shall say that $N$ is a compactification of $M$ corresponding to the boundary $Z$, and conversely, $Z$ is a boundary of $M$ corresponding to the compactification $N$. We recall also that a closed subset $Z$ of a space $X$ is said to be a $Z$-set if for each open $\operatorname{cover} \mathscr{U} \in \operatorname{cov}(X)$ there is a map $f: X \rightarrow X-Z \quad \mathscr{U}$-close to the identity map of $X)$. Having in mind a deep analogy between the theories of $\mu^{n+1}$-manifolds and $Q$-manifolds [1, 6-9] it is not surprising that the corresponding results are valid in the case of $\mu^{n+1}$. manifolds as well. However, it should be observed that the situation in the last case is much simpler. For example, the analogies of the above-described

Received by the editors September 23, 1992.

1991 Mathematics Subject Classification. Primary 57Q12, 57N99; Secondary 55P55.

Key words and phrases. $n$-homotopy equivalence, $n$-shape, Menger manifold. 
obstructions always vanish (Theorem 2.7). Moreover, we shall see that the different boundaries that can be put on a $\mu^{n+1}$-manifold constitute a whole $n$ shape class (Proposition 3.1), but, at the same time, every two compactifications of a $\mu^{n+1}$-manifold are equivalent in the sense of Chapman and Siebenmann (Proposition 3.2). Apparently one of the reasons for these differences is that the natural analogue of Wall's finiteness obstruction [15] vanishes in the $n$ homotopy category. In the arguments below, this last fact plays the key role although its proof is quite elementary and does not use anything except standard definitions and techniques from [15]. I am absolutely sure that this fact is well known to experts, but unfortunately I could not find any mention of it in the literature. For this reason the proof of this result (Proposition 1.2) is presented in $\S 1$.

\section{1. $n$-HOMOTOPY DOMINATION AND $n$-TAMENESS AT $\infty$}

Throughout the paper only locally compact metrizable spaces and continuous maps are considered. The letter $n$ denotes an arbitrary (but fixed) nonnegative integer. For information concerning $k$-dimensional Menger manifolds (briefly, $\mu^{k}$-manifolds) and $k$-shapes, see [1] and [7], respectively.

Two maps $f, g: X \rightarrow Y$ are said [5, 9] to be (properly) $n$-homotopic (notation: $f \stackrel{n}{\simeq} g$ and $f \stackrel{n}{\stackrel{n}{q}} g$ respectively) if for any (proper) map $h: Z \rightarrow X$ of any at most $n$-dimensional space $Z$ into $X$ the compostions $f h$ and $g h$ are (properly) homotopic in the usual sense. For the maps between at most $(n+1)$-dimensional $L C^{n}$-spaces the concept of (proper) $n$-homotopy coincides [6, Proposition 2.3] with the concept of (proper) $\mu$-homotopy introduced in [1]. It should be emphasized especially that as of 1941 Fox had a protoype of the notion of $n$-homotopy [11]. A (proper) map $f: X \rightarrow Y$ is called (proper) $n$-homotopy equivalence $[16,9]$ if it has a (proper) $n$-homotopy inverse, i.e., a (proper) map $g: Y \rightarrow X$ such that $g f \stackrel{n}{\simeq} \operatorname{id}_{X}$ and $f g \stackrel{n}{\simeq} \operatorname{id}_{Y}$ (respectively, $g f \underset{\bar{p}}{\stackrel{n}{\sim}} \mathrm{id}_{X}$ and $f g \underset{p}{\stackrel{n}{\sim}} \operatorname{id}_{Y}$ ). In this case we shall say that $X$ and $Y$ are (properly) $n$-homotopy equivalent. If only the first relation is satisfied then we shall say that $X$ is $n$-homotopy dominated by $Y$. Any proper $U V^{n}$-map between locally compact $L C^{n}$-spaces with at most $(n+1)$-dimensional range can serve as an example of proper $n$-homotopy equivalence. We recall that $X$ is a $U V^{n_{-}}$ compactum iff $X$ has a trivial $n$-shape. For information concerning $U V^{n}$-maps see [1] and [12].

Clearly every connected polyhedron is 0-homotopy equivalent to the onepoint space. Consequently any map between connected polyhedra is a 0 -homotopy equivalence. This simple observation together with the corresponding result of Whitehead [16, Theorem 2] give the following algebraic characterization of $n$-homotopy equivalences.

Proposition 1.1. A map $f: X \rightarrow Y$ between at most $(n+1)$-dimensional locally finite polyhedra is an n-homotopy equivalence iff it induces isomorphisms of homotopy groups of dimension $\leq n$, i.e., $f$ induces a bijection between the components of $X$ and $Y$ and a homomorphism $\pi_{k}\left(f^{\prime}\right): \pi_{k}\left(C_{X}\right) \rightarrow \pi_{k}\left(C_{Y}\right)$ is an isomorphism for each $k \leq n$ and each pair of components $C_{X} \subseteq X$ and $C_{Y} \subseteq Y$ with $f\left(C_{X}\right) \subseteq C_{Y}$, where $f^{\prime}: C_{X} \rightarrow C_{Y}$ denotes the restriction of $f$. 
Proposition 1.2. Let $M$ be at most $(n+1)$-dimensional locally finite polyhedron. Suppose that there exists at most $(n+1)$-dimensional finite polyhedron $K$ and two maps $f: M \rightarrow K$ and $g: K \rightarrow M$ such that $g f \stackrel{n}{\simeq} \operatorname{id}_{M}$. Then there exists at most $(n+1)$-dimensional finite polyhedron $T$, containing $K$ as a subpolyhedron, and an n-homotopy equivalence $h: T \rightarrow M$, extending $g$ such that $f$ is an $n$-homotopy inverse of $h$.

Proof. It suffices to consider only connected polyhedra. Consequently, the case $n=0$ is trivial. If $n=1$, then, by the assumptions, $\pi_{1}(g): \pi_{1}(K) \rightarrow \pi_{1}(M)$ is an epimorphism and $\operatorname{Ker}\left(\pi_{1}(g)\right)$ is a finitely generated group. Select finitely many generators of $\operatorname{Ker}\left(\pi_{1}(g)\right)$, and use them to attach 2-cells to $K$ and to extend $g$ over these cells. In this way we obtain a 2-dimensional finite polyhedron $T$, containing $K$ as a subpolyhedron, and a map $h: T \rightarrow M$, extending $g$, which induces an isomorphism of fundamental groups. By Proposition 1.1, $h$ is a 1 -homotopy equivalence.

Assume, by induction, that the proposition is already proved in the cases $n \leq m, m \geq 1$, and consider the case $n=m+1$. Without loss of generality we can suppose that $f\left(M^{i}\right) \subseteq K^{i}$ and $g\left(K^{i}\right) \subseteq M^{i}$ for each $i \leq m+1$. Since $g f \stackrel{m+1}{\simeq} \mathrm{id}_{M}$ it follows easily that $g f / M^{m+1} \stackrel{m}{\simeq} \mathrm{id}_{M^{m+1}}$. By the inductive hypothesis, there are an $(m+1)$-dimensional finite polyhedron $R$, containing $K^{m+1}$ as a subpolyhedron, and an $m$-homotopy equivalence $r: R \rightarrow M^{m+1}$, extending $g / K^{m+1}$. Sewing together the polyhedra $K$ and $R$ along naturally embedded copies of $K^{m+1}$ we obtain the $(m+2)$-dimensional finite polyhedron $L$, containing $K$ and $R$ as subpolyhedra, and the map $s: L \rightarrow M$ which coincides with $g$ on $K$ and with $r$ on $R$, whence $s f=g f \stackrel{m+1}{\simeq} \operatorname{id}_{M}$ and $f s / L^{m+1}=f r \stackrel{m}{\simeq} \operatorname{id}_{L^{m+1}}$. By these conditions, we conclude that $\pi_{i}(s): \pi_{i}(L) \rightarrow$ $\pi_{i}(M)$ is an isomorphism for each $i \leq m$ and an epimorphism for $i=m+1$. One can easily verify that in this situation $\operatorname{Ker}\left(\pi_{m+1}(s)\right)$ is a finitely generated $\mathbb{Z}\left(\pi_{1}(L)\right)$-module. Select $\mathbb{Z}\left(\pi_{1}(L)\right)$-generators for $\operatorname{Ker}\left(\pi_{m+1}(s)\right)$ and use them to attach $(m+2)$-cells to $L$ and to extend $s$ over these cells. Let $T$ denote the resulting $(m+2)$-dimensional finite polyhedron, containing $L$ as a subpolyhedron, and $h: T \rightarrow M$ the corresponding extension of $s$. Then $\pi_{i}(h)$ is an isomorphism for each $i \leq m+1$. Again, by Proposition 1.1, $h$ is an $(m+1)$-homotopy equivalence. This performs the inductive step and finishes the proof.

Corollary 1.3. If a $\mu^{n+1}$-manifold $M$ is $n$-homotopy dominated by at most $(n+1)$-dimensional $L C^{n}$-compactum, then $M$ is $n$-homotopy equivalent to a compact $\mu^{n+1}$-manifold.

Proof. By the triangulation theorem for $\mu^{n+1}$-manifolds [7], there exists a proper $U V^{n}$-retraction, $r: M \rightarrow P$ onto some locally finite polyhedron. Note that, by [8, Proposition 1.4] $r$ is an $n$-homotopy equivalence. Let $X$ be at most $(n+1)$-dimensional $L C^{n}$-compactum which $n$-homotopy dominates $M$. By [8, Proposition 1.5], $X$ is $n$-homotopy equivalent to an at most $(n+1)$ dimensional finite polyhedron $L$. Consequently, $L$-homotopy dominates $P$. By Proposition 1.2 , there is an $(n+1)$-dimensional finite polyhedron $T$ (containing $L$ ) $n$-homotopy equivalent to $P$. Consider now a $U V^{n}$-surjection $f: N \rightarrow T$ of some compact $\mu^{n+1}$-manifold $N$ onto $T$ [10]. It only remains to note that $M$ and $N$ are $n$-homotopy equivalent. The proof is finished. 
The following concept is an analogue of the well-known notion of tameness at $\infty$.

Definition 1.4. A space $X$ is said to be $n$-tame at $\infty$ if for each compactum $A \subseteq X$ there exists a larger compactum $B \subseteq X$ such that the inclusion $X-B \rightarrow$ $X-A$ factors up to $n$-homotopy through an at most $(n+1)$-dimensional finite polyhedron.

Proposition 1.5. If a $\mu^{n+1}$-manifold is $n$-tame at $\infty$, then it is $n$-homotopy equivalent to a compact $\mu^{n+1}$-manifold.

Proof. Fix a proper $U V^{n}$-retraction $r: M \rightarrow P$ of a given $\mu^{n+1}$-manifold $M$ onto some $(n+1)$-dimensional polyhedron $P$ [7]. It follows from the wellknown properties of proper $U V^{n}$-maps [12] that $P$ is $n$-tame at $\infty$ as well. Using [6, Proposition 2.2] instead of the usual homotopy extension theorem and repeating the proof of Lemma 5.1 from [5] we can conclude that $P$ is $n$-homotopy dominated by an at most $(n+1)$-dimensional finite polyhedron. Corollay 1.3 finishes the proof.

\section{The MAIN RESULT}

Let us recall that a $Q$-manifold $M$ lying in a larger $Q$-manifold $N$ is said to be clean in $N$ [5] if $M$ is closed in $N$ and the topological frontier of $M$ in $N$ is collared both in $M$ and $N-\operatorname{Int} M$. By obvious dimensional reasons we cannot define directly the corresponding notion in the case of $\mu^{n+1}$-manifolds. Nevertheless, the following notion will be useful for us.

Definition 2.1. A $\mu^{n+1}$-manifold $M$ lying in a $\mu^{n+1}$-manifold $N$ is said to be $n$-clean (in $N$ ) provided that $M$ is closed in $N$ and there' exists a closed subspace $\delta(M)$ of $M$ such that the following conditions are satisfied:

(i) $\delta(M)$ is a $\mu^{n+1}$-manifold;

(ii) $(N-M) \cup \delta(M)$ is a $\mu^{n+1}$-manifold;

(iii) $\delta(M)$ is a $Z$-set in $M$;

(iv) $\delta(M)$ is a $Z$-set in $(N-M) \cup \delta(M)$; and

(v) $M-\delta(M)$ is open in $N$.

Let us indicate the standard situation when $n$-clean submanifolds arise naturally. Suppose that $L$ is a submanifold of a combinatorial PL-manifold $P$. Fix a $U V^{n}$-map $f: N \rightarrow P$ of some $\mu^{n+1}$-manifold $N$ onto $P$ constructed in [10, Theorem 1.3] (see also [9, Theorem 1.6]). Using the properties of $f$ it is easy to see that $M=f^{-1}(L)$ is an $n$-clean submanifold of $N$ with $\delta(M)=f^{-1}(\partial L)$. Generally speaking $f$ is not an open map and consequently $\delta(M)$ does not necessarily coincide with the topological frontier of $M$ in $N$.

Lemma 2.2. Let $N$ be a $\mu^{n+1}$-manifold which is $n$-tame at $\infty$. Suppose that $M$ is a compact and $n$-clean submanifold of $N$. Then the $\mu^{n+1}$-manifold $(N-M) \cup \delta(M)$ is $n$-homotopy equivalent to a compact $\mu^{n+1}$-manifold.

Proof. By Proposition 1.5, it suffices to show that a $\mu^{n+1}$-manifold $(N-M) \cup$ $\delta(M)$ is $n$-tame at $\infty$. Let $A$ be a compact subspace of $(N-M) \cup \delta(M)$. Clearly, $K_{1}=A \cup M$ is compact. Since $N$ is $n$-tame at $\infty$, there exists a compactum $K_{2}$ such that $K_{1} \subseteq K_{2} \subseteq N$ and the inclusion $N-K_{2} \rightarrow N-$ $K_{1}$ factors up to $n$-homotopy through an at most $(n+1)$-dimensional finite 
polyhedron. Let $B=((N-M) \cup \delta(M)) \cap K_{2}$. Clearly $B$ is compact and $A \subseteq B$. Note that $((N-M) \cup \delta(M))-B=N-K_{2}$ and $N-K_{1} \subseteq((N-M) \cup \delta(M))-A$. Consequently, the inclusion $((N-M) \cup \delta(M))-B \rightarrow((N-M) \cup \delta(M))-$ $A$ factors up to $n$-homotopy through an at most $(n+1)$-dimensional finite polyhedron. Hence, $(N-M) \cup \delta(M)$ is $n$-tame at $\infty$. The proof is finished.

Lemma 2.3. Each $\mu^{n+1}$-manifold $M$ can be written as a union $M=\bigcup\left(M_{i}\right.$ : $i \in \omega)$ such that all $M_{i}$ 's are compact and $n$-clean and $M_{i} \subseteq M_{i+1}-\delta\left(M_{i+1}\right)$, $i \in \omega$.

Proof. It suffices to show that for each compactum $K \subseteq M$ there exists a compact and $n$-clean $M_{1} \subseteq M$ such that $K \subseteq M_{1}-\delta\left(M_{1}\right)$. As in [7] fix a proper $U V^{n}$-surjection $g: M \rightarrow X$, where $X$ is a $Q$-manifold. By [5] there exists a compact and clean $Y \subseteq X$ such that $g(K) \subseteq \operatorname{Int}_{X}(Y)$. By the relative triangulation theorem for $Q$-manifolds [4, Lemma 37.1], there exists a polyhedron $P$ which can be written as a union of two subpolyhedra $P_{1}$ and $P_{2}$ such that $X=P \times Q, Y=P_{1} \times Q, X-\operatorname{Int}_{X}(Y)=P_{2} \times Q$, and $\operatorname{Bd}_{X}(Y)=\left(P_{1} \cap P_{2}\right) \times Q$. Note also that the subpolyhedron $P_{1} \cap P_{2}$ is a $Z$-set both in $P_{1}$ and $P_{2}$.

By [9-10], there exists a proper $U V^{n}$-surjection $f: N \rightarrow P$ of some $\mu^{n+1}$ manifold $N$ onto $P$ satisfying the following two conditions:

(a) If $L$ is a subpolyhedron of $P$ then $f^{-1}(L)$ is a $\mu^{n+1}$-manifold,

(b) If $L$ is a subpolyhedron of $P$ and $A$ is a $Z$-set in $L$ then $f^{-1}(Z)$ is a $Z$-set in $f^{-1}(L)$.

Consequently we have two proper $U V^{n}$-surjections $f: N \rightarrow P$ and $\pi_{p} g: M$ $\rightarrow P\left(\pi_{P}: P \times Q \rightarrow P\right.$ denotes the natural projection) of two $\mu^{n+1}$-manifolds onto the polyhedron $P$. Consider an open cover $\mathscr{U}=\left\{P-\pi_{P} g(K), \operatorname{Int}_{P}\left(P_{1}\right)\right\}$ of $P$. By [1, Remark 5.1.1], there exists a homeomorphism $h: M \rightarrow N$ such that the compositions $\pi_{P} g$ and $f h$ are $\mathscr{U}$-close. Let $M_{1}=h^{-1} f^{-1}\left(P_{1}\right)$ and $\delta\left(M_{1}\right)=h^{-1} f^{-1}\left(P_{1} \cap P_{2}\right)$. By the properties of the map $f, M_{1}$ is compact and $n$-clean. It only remains to note that $K \subseteq M_{1}-\delta\left(M_{1}\right)$. This finishes the proof.

The following proposition is a direct consequence of the chacterization theorem for $\mu^{n+1}$-manifolds [1].

Proposition 2.4. Let a space $M$ be a union of two closed subspaces $M_{1}$ and $M_{2}$. If $M_{1}, M_{2}$, and $M_{0}=M_{1} \cap M_{2}$ are $\mu^{n+1}$-manifolds and $M_{0}$ is a $Z$-set both in $M_{1}$ and $M_{2}$, then $M$ is a $\mu^{n+1}$-manifold.

Proof. By [1], it suffices to show that for any map $f: X \rightarrow M$ of any at most $(n+1)$-dimensional compactum $X$ into $M$ and any open cover $\mathscr{U} \in \operatorname{cov}(M)$ there exists an embedding $g: X \rightarrow M \mathscr{U}$-close to $f$. Let us consider the case when $f(X) \cap M_{i} \neq \varnothing$ for each $i=0,1,2$. All other possible cases are trivial. By [6, Proposition 2.1], there exists an open cover $\mathscr{V} \in \operatorname{cov}(M)$ refining $\mathscr{U}$ such that the following condition holds:

$(*)_{n}$ for any at most $(n+1)$-dimensional compactum $B$, its closed subspace $A$, and any two $\mathscr{V}$-close maps $\alpha_{1}, \alpha_{2}: A \rightarrow M$ such that $\alpha_{1}$ has an extension $\beta_{1}: B \rightarrow M$, it follows that $\alpha_{2}$ also has an extension $\beta_{2}: B \rightarrow M$ which is $\mathscr{U}$-close to $\beta_{1}$.

Let $X_{i}=f^{-1}\left(M_{i}\right), i=0,1,2$. Since $M_{0}$ is a $\mu^{n+1}$-manifold, there is a $Z$-embedding $g_{0}: X_{0} \rightarrow M_{0}$ such that $g_{0}$ and $f / X_{0}$ are $\mathscr{V}$-close. By $(*)_{n}$, there is an extension $h: X \rightarrow M$ of $g_{0}$ such that $h$ and $f$ are $\mathscr{V}$-close. Since 
$M_{0}$ is a $Z$-set both in $M_{1}$ and $M_{2}$ we conclude that $g_{0}\left(X_{0}\right)$ is a $Z$-set both in $M_{1}$ and $M_{2}$. Consequently, by [1, Chapter 6, the $Z$-set Approximation Theorem] for each $i=1,2$, there is a $Z$-embedding $g_{i}: X_{i} \rightarrow M_{i}$ such that $g_{i} / X_{0}=g_{0}$ and $g_{i}$ is $\mathscr{U}$-close to $h / X_{i}$. At the same time without loss of generality we can assume that one of these maps, say $g_{1}$, has the following property: $g_{1}\left(X_{1}-X_{0}\right) \cap M_{0}=\varnothing$ (we once again use the fact that $M_{0}$ is a $Z$-set in $\left.M_{1}\right)$. Then the map $g$ coinciding with $g_{i}$ on $X_{i} \quad(i=1,2)$ is an embedding. It only remains to note that $g$ and $f$ are $\mathscr{U}$-close. The proof is finished.

Lemma 2.5. If a $\mu^{n+1}$-manifold $M$ is $n$-tame at $\infty$, then we can write $M=$ $\bigcup\left(M_{i}: i \in \omega\right)$ such that all $M_{i}$ 's are compact and n-clean, $M_{i} \subseteq M_{i+1}-\delta\left(M_{i+1}\right)$ and the inclusion $\delta\left(M_{i}\right) \rightarrow\left(M_{i+1}-M_{i}\right) \cup \delta\left(M_{i}\right)$ is n-homotopy equivalence for each $i \in \omega$.

Proof. Choose any compact and $n$-clean submanifold $A$ of $M$. By Lemma 2.3, it will suffice to find a compact and $n$-clean submanifold $B$ of $M$ such that $A \subseteq B-\delta(B)$ and the inclusion $\delta(B) \rightarrow(M-B) \cup \delta(B)$ is an $n$-homotopy equivalence. By Lemma 2.2, the $\mu^{n+1}$-manifold $(M-A) \cup \delta(A)$ is $n$-homotopy equivalent to some compact $\mu^{n+1}$-manifold $X$. Fix the corresponding $n$ homotopy equivalence $\psi_{1}:(M-A) \cup \delta(A) \rightarrow X$ and its $n$-homotopy inverse $\varphi_{1}: X \rightarrow(M-A) \cup \delta(A)$. By [1, Theorem 2.3.8] and [6, Lemma 2.1 and Proposition 2.1], there is a map $\psi_{2}:(M-A) \cup \delta(A) \rightarrow X$ such that $\psi_{2} / \delta(A): \delta(A) \rightarrow X$ is a $Z$-embedding and $\psi_{2}$ is as close to $\psi_{1}$ as we wish. Similarly, there is a $Z$-embedding $\varphi_{2}: X \rightarrow(M-A) \cup \delta(A)$ which is as close to $\varphi_{1}$ as we wish. Particularly, we can assume that $\psi_{2}$ and $\varphi_{2}$ are $n$-homotopy equivalences. If $\psi_{2}$ and $\varphi_{2}$ were chosen sufficiently close to $\psi_{1}$ and $\varphi_{1}$, respectively, then, by the $Z$-set unknotting theorem [1], there exists a homeomorphism $h:(M-A) \cup \delta(A) \rightarrow(M-A) \cup \delta(A)$ which extends the homeomorphism $\varphi_{2} \psi_{2} / \delta(A): \delta(A) \rightarrow \varphi_{2} \psi_{2}(\delta(A))$ and which is sufficiently close to the identity map of $(M-A) \cup \delta(A)$. Particularly, we can assume that $h$ is $n$-homotopic to $\operatorname{id}_{(M-A) \cup \delta(A)}$. Then an $n$-homotopy equivalence $\varphi=$ $h^{-1} \varphi_{2}: X \rightarrow(M-A) \cup \delta(A)$, is a $Z$-embedding and $\delta(A) \subseteq \varphi(X) \equiv Y$. Since $Y$ is a compact $\mu^{n+1}$-manifold there exists [7] a $U V^{n}$-retraction $s: Y \rightarrow K$ onto a finite $(n+1)$-dimensional polyhedron $K$. Similarly fix a proper $U V^{n}$-retraction $r:(M-A) \cup \delta(A) \rightarrow T$, where $T$ is a polyhedron. Let $i: Y \rightarrow(M-A) \cup \delta(A)$ denote the inclusion map and $j: K \rightarrow Y$ be a section of $s$ (i.e., $s j=\operatorname{id}_{K}$ ). Note that $i$ is an $n$-homotopy equivalence. Let $p: K \rightarrow T$ be a PL-map homotopic to the compostion $r i j$. Form the mapping cylinder $M(p) \equiv P$ of the map $p$. Let us recall [4] that this is a space formed from the disjoint union $(K \times[0,1]) \oplus T$, by identifying $(k, 1)$ with $p(k), k \in K$. At the same time we identify $K$ with $K \times\{0\}$. Since $p$ is a PL-map, $K \times\{0\}$ and $T$ are subpolyhedra of the polyhedron $P$. Let $c: P \rightarrow T$ be the collapse to the base, i.e., the natural retraction defined by sending $(k, t)$ to $p(k)$ for each $(k, t) \in K \times[0,1]$. Obviously $c$ is a proper CE-map that is a proper homotopy equivalence. Fix a proper $U V^{n}$-surjection $f: N \rightarrow P$ of some $\mu^{n+1}$-manifold onto $P$ satisyfying the conditions (a) and (b) in the proof of Lemma 2.3. Compact $\mu^{n+1}$ manifolds $Y$ and $N_{1}=f^{-1}(K \times\{0\})$ admit $U V^{n}$-surjections $s: Y \rightarrow K \times\{0\}$ and $f / N_{1}: N_{1} \rightarrow K \times\{0\}$ onto the same polyhedron. Consequently, by [1], there exists a homeomorphism $g_{1}: Y \rightarrow N_{1}$ such that $f g_{1} \stackrel{n}{\sim} s$. Similarly, we 
have two proper $U V^{n}$-surjections $r:(M-A) \cup \delta(A) \rightarrow T$ and $c f: N \rightarrow T$. As above there is a homeomorphism $g_{2}:(M-A) \cup \delta(A) \rightarrow N$ such that $c f g_{2} \underset{p}{\stackrel{n}{\sim}} r$. By the construction and the corresponding properties of $U V^{n}$-surjections [12], we have $c f g_{1} \stackrel{n}{\simeq} c s=p s \simeq r i j s \stackrel{n}{\simeq} r i \stackrel{n}{\simeq} c f g_{2} i$. Since $c f$ is a proper $n$ homotopy equivalence, we conclude that $g_{1}: Y \rightarrow N$ and $g_{2} / Y: Y \rightarrow N$ are $n$-homotopic. Consider the homeomorphism $\alpha=g_{1} g_{2}^{-1} / g_{2}(Y): g_{2}(Y) \rightarrow N_{1}$. Clearly, $\alpha \stackrel{n}{\simeq} g_{2} g_{2}^{-1} / g_{2}(Y)=\operatorname{id}_{g_{2}(Y)}$. By the properties of the map $f, N_{1}$ is a $Z$-set in $N$. Note also that, by our construction, $g_{2}(Y)$ is a $Z$-set in $N$ as well. Using the $Z$-set unknotting theorem [1] we can find a homeomorphism $G: N \rightarrow$ $N$ extending $\alpha$. Let $H=G g_{2}$. Note that $H(Y)=G g_{2}(Y)=\alpha g_{2}(Y)=N_{1}$. Finally, let $B=A \cup H^{-1}\left(f^{-1}\left(K \times\left[0,2^{-1}\right]\right)\right)$ and $\delta(B)=H^{-1}\left(f^{-1}\left(K \times\left\{2^{-1}\right\}\right)\right)$. It follows from the properties of the map $f$ and Proposition 2.4 that $B$ is a compact and $n$-clean submanifold of $M, A \subseteq B-\delta(B)$ and the inclusion $\delta(B) \rightarrow(M-B) \cup \delta(B)$ is an $n$-homotopy equivalence (note that the map $p$ and consequently the inclusion $K \times\left\{2^{-1}\right\} \rightarrow P-\left(K \times\left[0,2^{-1}\right]\right)$ are $n$-homotopy equivalences). This finishes the proof.

Lemma 2.6. Let a $\mu^{n+1}$-manifold $M$ be a Z-set in a compact $\mu^{n+1}$-manifold $N$. If the inclusion $i: M \rightarrow N$ is an $n$-homotopy equivalence, then there exists a $U V^{n}$-surjection of $N$ onto $M$.

Proof. Let $j: N \rightarrow M$ be an $n$-homotopy inverse of $i$. By [1, Theorem 2.8.6], there is a homeomorphism $h: N \rightarrow M$ such that $h \stackrel{n}{\simeq} j$. Then $h i \stackrel{n}{\simeq} j i \stackrel{n}{\simeq} \mathrm{id}_{M}$. Consequently, by [1, Proposition 5.1.2], there is a $U V^{n}$-retraction $r: N \rightarrow M$ such that $r i=\mathrm{id}_{M}$. The proof is finished.

The following theorem is the main result of this paper and gives a characterization of $\mu^{n+1}$-manifolds with boundaries.

Theorem 2.7. A $\mu^{n+1}$-manifold admits a boundary iff it is $n$-tame at $\infty$.

Proof. Let $M$ be a $\mu^{n+1}$-manifold which is $n$-tame at $\infty$. By Lemma 2.5, we can represent $M$ as a union $M=\bigcup\left\{M_{i}: i \in \omega\right\}$ such that all the $M_{i}$ 's are compact and $n$-clean, $M_{i} \subseteq M_{i+1}-\delta\left(M_{i+1}\right)$ and the inclusion $\delta\left(M_{i}\right) \rightarrow$ $\left(M_{i+1}-M_{i}\right) \cup \delta\left(M_{i}\right)$ is $n$-homotopy equivalent for each $i \in \omega$. By Lemma 2.6, for each $i \in \omega$ there exists a $U V^{n}$-retraction $f_{i}:\left(M_{i+1}-M_{i}\right) \cup \delta\left(M_{i}\right) \rightarrow \delta\left(M_{i}\right)$. Let a $U V^{n}$-retraction $r_{i}: M_{i+1} \rightarrow M_{i}$ coincide with $f_{i}$ on $M_{i+1}-M_{i}$ and with identity on $M_{i}, i \in \omega$. Then we have an inverse sequence $S=\left\{M_{i}, r_{i}\right\}$ consisting of compact $\mu^{n+1}$-manifolds and $U V^{n}$-retractions. By [1, Corollary 4.3.2], $r_{i}$ is a near-homeomorphism for each $i \in \omega$. By [3, Theorem 4], each limit projection of the spectrum $S$ is a near-homeomorphism as well. Consequently, $N=\lim S$, being homeomorphic to $M_{0}$, is a compact $\mu^{n+1}$ manifold. Since $\delta\left(M_{i}\right)$ is a $Z$-set in $M_{i}$ for each $i \in \omega$, we conclude that the subset $Z=\lim \left\{\delta\left(M_{i+1}\right), r_{i} / \delta\left(M_{i+1}\right)\right\}$ is a $Z$-set in $N$. It only remains to note that $N \overleftarrow{-Z}$ is naturally homeomorphic to $M$.

Conversely, suppose that a $\mu^{n+1}$-manifold $M$ admits a boundary. This means that there are a compact $\mu^{n+1}$-manifold $N$ and a $Z$-set $Z$ in $N$ such that $M=N-Z$. Let us show that $M$ is $n$-tame at $\infty$. Let $A$ be a compact subspace of $M$. By the proof of Lemma 2.3, there exists a compact and $n$-clean submanifold $B$ of $M$ such that $A \subseteq B-\delta(B)$. It suffices to show that 
$(M-B) \cup \delta(B)$ is $n$-homotopy equivalent to an at most $(n+1)$-dimensional finite poyhedron. Indeed, it is easy to see that $(M-B) \cup \delta(B)$ is $n$-homotopy equivalent to a compact $\mu^{n+1}$-manifold $(N-B) \cup \delta(B)$. It only remains to note that, by [8, Proposition 1.5], each compact $\mu^{n+1}$-manifold is $n$-homotopy equivalent to an at most $(n+1)$-dimensional finite polyhedron. The proof is finished.

In [5] Whitehead's example of contractible open subspace $W$ of $R^{3}$ (which is not tame at $\infty$ ) was used to construct a $Q$-manifold without boundary. The same example can be used in our case as well. Indeed, consider any $\mu^{4}$-manifold $M$ admitting a proper $U V^{3}$-retraction onto $W$. Then $M$ is not 3 -tame at $\infty$ and, by Theorem 2.7 , does not admit a boundary.

Theorem 2.7 can be used in somewhat different direction as well. Consider the problem of topological characterization of the space $\mu^{n+1}-\{\mathrm{pt}\}$. This space is stable in the sense of [9] and there are many other reasons indicating that it deserves special attention. Of course, $\mu^{n+1}-\{\mathrm{pt}\}$ is a $\mu^{n+1}$-manifold, and hence, in the light of Bestvina's results [1], we have to characterize this space only among $\mu^{n+1}$-manifolds.

Corollary 2.8. Let $X$ be a $\mu^{n+1}$-manifold satisfying the following conditions:

(i) $X$ is n-tame at $\infty$;

(ii) $X$ is $L C^{n}$ at $\infty$; and

(iii) $X \in C^{n}$.

Then $X$ is homeomorphic to $\mu^{n+1}-\{\mathrm{pt}\}$.

Proof. By Theorem 2.7 and (i) we can write $X=N-Z$, where $N$ is a compact $\mu^{n+1}$-manifold and $Z$ is a $Z$-set in $N$. By (iii) and [1], $N$ is a copy of $\mu^{n+1}$. By (ii), $Z$ is a $U V^{n}$-compactum. But $U V^{n}$-compacta have trivial $n$-shape [6]. Consequently, by [6], $X=\mu^{n+1}-Z \approx \mu^{n+1}-\{$ pt $\}$.

\section{BOUNDARIES AND COMPACTIFICATIONS}

In this section we present two propositions which give classifications of boundaries and compactifications of $\mu^{n+1}$-manifolds.

Proposition 3.1. If a compactum $X$ is a boundary for a $\mu^{n+1}$-manifold $M$, then a compactum $Y$ is also a boundary for $M$ iff $\operatorname{dim} Y \leq n+1$ and $n-\operatorname{Sh}(Y)=$ $n-\operatorname{Sh}(X)$.

Proof. Let $X$ and $Y$ be boundaries for $M$. We wish to show that $n-S h(Y)=$ $n-S h(X)$. Let $N=M \cup X$ and $T=M \cup Y$ be corresponding compactifcations of $M$ that are $\mu^{n+1}$-manifolds. Fix a $Z$-embedding $f: N \rightarrow N$ such that $f \stackrel{n}{\simeq} \operatorname{id}_{N}$ and $f(N) \cap X=\varnothing$. Embed $f(N)$ in $\mu^{n+1}$ as a $Z$-set (see [1]). Identifying $f(N)$ with the copy of $f(N)$ in $\mu^{n+1}$ we obtain a compactum $N_{1}=N \cup \mu^{n+1}$. By Proposition 2.4, $N_{1}$ is a $\mu^{n+1}$-manifold. It is easy to verify that in our situation $N_{1} \in C^{n}$ and, hence, by [1], $N_{1}$ is homeomorphic to $\mu^{n+1}$.

By our assumption, there is a homeomorphism $h: N-X \rightarrow T-Y$. Embed $h(f(N))$ into $\mu^{n+1}$ as a $Z$-set and identify $h(f(N))$ with the copy of $h(f(N))$ in $\mu^{n+1}$. As above a $\mu^{n+1}$-manifold $T_{1}=T \cup \mu^{n+1}$ is homeomorphic to $\mu^{n+1}$. Evidently there is a homeomorphism $H: N_{1} \rightarrow T_{1}$ such that $H\left(N_{1}-X\right)=$ $T_{1}-Y$. Consequently, by the main result of [6], $n-\operatorname{Sh}(Y)=n-\operatorname{Sh}(X)$. 
Conversely, let $X$ be a boundary for $M$ and $Y$ be an at most $(n+1)$ dimensional compactum such that $n-S h(Y)=n-S h(X)$. Let $N=M \cup X$ be a compactification of $M$ corresponding to $X$. Form $N_{1}=N \cup \mu^{n+1}$ as above. Since $\operatorname{dim} Y \leq n+1$, we can assume that $Y$ is a $Z$-set in $N_{1}$. Since $n-S h(Y)=n-S h(X)$, it follows from [6] that there is a homeomorphism $h: N_{1}-X \rightarrow N_{1}-Y$. Let us show that $T=h(N-X) \cup Y$ is a compact $\mu^{n+1}$-manifold and $Y$ is a $Z$-set in $T$. Since $N$ is an $L C^{n}$-compactum, so is $h(f(N))$. Hence, there is a retraction $s: S \rightarrow h(f(N))$, where $S$ is an open neighbourhood of $h(f(N))$ in $h\left(\mu^{n+1}\right)$. Let $G=S \cup T$ and the map $r: G \rightarrow T$ coincide with $S$ on $S$ with $\operatorname{id}_{T}$ on $T$. Clearly $G$ is an open neighbourhood of $T$ in $N_{1}$ and $r$ is a retraction. This shows that $T$ is an $L C^{n}$-compactum. In order to prove that $Y$ is a $Z$-set in $T$, fix an arbitrary open cover $\mathscr{U} \in \operatorname{cov}(T)$ and let $\mathscr{V}=\left\{r^{-1}(U): U \in \mathscr{U}\right\} \cup\left\{N_{1}-T\right\}$. Clearly $\mathscr{V}$ is an open cover of $N_{1}$. Since $Y$ is a $Z$-set in $N_{1}$, there is a map $g: N_{1} \rightarrow N_{1}$ such that $g\left(N_{1}\right) \cap Y=\varnothing$ and which is $\mathscr{V}$-close to the identy map of $N_{1}$. Note that $g(T) \subseteq G$ and consequently the composition $r g / T: T \rightarrow T$ is well defined. An easy verification shows that $r g / T$ is $\mathscr{U}$-close to $\operatorname{id}_{T}$ and $r g(T) \cap Y=\varnothing$. Thus, $Y$ is a $Z$-set in $T$. Finally note that $T$ is an $(n+1)$-dimensional $L C^{n}$ compactum and contains a $Z$-set $Y$ complement $T-Y=h(N-X)$ of which is a $\mu^{n+1}$-manifold. This allows us to conclude that $T$ is a $\mu^{n+1}$-manifold itself. The proof is finished.

Let us recall [5] that two compactifications $N$ and $T$ of the same space $M$ are said to be equivalent if for every compactum $A \subseteq M$ there is a homeomorphism of $N$ onto $T$ fixing $A$ pointwise.

Of course, if $\mu^{n+1}$-manifolds $N$ and $T$ are compactifications of a $\mu^{n+1}$ manifold $M$, then the inclusions $M \rightarrow N$ and $M \rightarrow T$ are $n$-homotopy equivalences (because, $N-M$ and $T-M$ are $Z$-sets in $N$ and $T$, respectively). Consequently, $N$ and $T$ are homeomorphic as $n$-homotopy equivalent compact $\mu^{n+1}$-manifolds [1]. We show now that $N$ and $T$ are equivalent even in the sense of Chapman-Siebenmann.

Proposition 3.2. Every two compactifications of a $\mu^{n+1}$-manifold are equivalent in the sense of Chapman-Siebenmann.

Proof. We keep the above notation. Let $A$ be a compact subspace of $M$. Choose a compact and $n$-clean submanifold $K$ of $M$ such that $A \subseteq K$ (Lemma 2.3). It follows from the definition that $N_{1}=(N-K) \cup \delta(K)$ and $T_{1}=(T-K) \cup \delta(K)$ are compact $\mu^{n+1}$-manifolds. Let $X=N-M$ and $Y=T-M$. Then $X$ and $Y$ are $Z$-sets in $N_{1}$ and $T_{1}$, respectively, and $N_{1}-X=T_{1}-Y=(M-K) \cup \delta(K)$. Consequently, the inclusions $i: N_{1}-X \rightarrow N_{1}$ and $j: T_{1}-Y \rightarrow T_{1}$ are $n$-homotopy equivalences. Let $s: N_{1} \rightarrow N_{1}-X$ be an $n$-homotopy inverse of $i$. Then $j s: N_{1} \rightarrow T_{1}$ is an $n$-homotopy equivalence. Consequently, by [1], there is a homeomorphism $h: N_{1} \rightarrow T_{1} n$-homotopic to the composition $j s$. Using the $Z$-set unknotting theorem we can assume without loss of generality that $h / \delta(K)=\mathrm{id}$. The desired homeomorphism $H: N \rightarrow T$ can be defined as one which coincides with $h$ on $N_{1}$ and with identity on $K$. The proof is finished. 


\section{ACKNOWLEDGMENTS}

Finally the author expresses his sincere appreciation to the referee for many helpful suggestions and remarks.

\section{REFERENCES}

1. M. Bestvina, Characterizing $k$-dimensional universal Menger compacta, $\mathrm{Ph} . \mathrm{D}$. thesis, Univ. of Tennessee, Knoxville, 1984.

2. W. Browder, J. Levine, and G. R. Livesay, Finding a boundary for an open manifold, J. Math. 87 (1965), 1017-1028.

3. M. Brown, Some applications of an approximation theorem for inverse limits, Proc. Amer. Math. Soc. 11 (1960), 478-481.

4. T. A. Chapman, Lectures on Hilbert cube manifolds, CBMS Regional Conf. Ser. in Math., vol. 28, Amer. Math. Soc., Providence, RI, 1976.

5. T. A. Chapman and L. C. Siebenmann, Finding a boundary for a Hilbert cube manifold, Acta Math. 137 (1976), 171-208.

6. A. Chigogidze, Compacta lying in the n-dimensional universal Menger compactum and having homeomorphic complements in it, Mat. Sb. 133 (1987), 481-496.

7. _ Theory of $n$-shape, Uspekhi Mat. Nauk 44 (1989), 117-140.

8. $\longrightarrow$, n-shapes and n-cohomotopy groups of compacta, Mat. Sb. 180 (1989), 322-335.

9. __ Classification theorem for Menger manifolds, Proc. Amer. Math. Soc. 116 (1992), 825-832.

10. —_ UV $V^{n}$-equivalence and n-equivalence, Topology Appl. 45 (1992), 283-291.

11. R. H. Fox, On the Lusternik-Schnirelman category, Ann. of Math. 42 (1941), 333-370.

12. R. C. Lacher, Cell-like mappings and their generalizations, Bull. Amer. Math. Soc. 83 (1977), 495-552.

13. S. Metcalf, Finding a boundary for a Hilbert cube manifold bundle, Pacific J. Math. 120 (1985), 153-178.

14. L. C. Siebenmann, The obstruction to finding a boundary for an open manifold of dimension greater than five, Doctoral dissertation, Princeton Univ., Princeton, NJ, 1965.

15. C. T. C. Wall, Fitness conditions for CW-complexes, Ann. of Math. 81 (1965), 56-69.

16. J. H. C. Whitehead, Combinatorial homotopy. I, Bull. Amer. Math. Soc. 55 (1949), 213-245.

Department of Mathematics and Statistics, University of Saskatchewan, Saskatoon, Saskatchewan, Canada S7N 0W0

E-mail address: chigogid@snoopy.usask.ca 